\title{
Secreted glutamic protease rescues aspartic protease Pep deficiency in Aspergillus fumigatus during growth in acidic protein medium
}

\section{Correspondence \\ Michel Monod \\ Michel.Monod@chuv.ch}

Received 27 January 2011

Revised 21 February 2011

Accepted 22 February 2011
Dev Sriranganadane, ${ }^{1}$ Utz Reichard ${ }^{2}$ Karine Salamin, ${ }^{1}$ Marina Fratti, ${ }^{1}$ Olivier Jousson, ${ }^{3}$ Patrice Waridel, ${ }^{4}$ Manfredo Quadroni, ${ }^{4}$ Jean-Marc Neuhaus ${ }^{5}$ and Michel Monod ${ }^{1}$

\author{
${ }^{1}$ Department of Dermatology, Centre Hospitalier Universitaire Vaudois, 1011 Lausanne, \\ Switzerland \\ ${ }^{2}$ Department of Medical Microbiology and National Reference Centre for Systemic Mycoses, \\ University Hospital of Göttingen, Germany \\ ${ }^{3}$ Centre for Integrative Biology (CIBIO), University of Trento, Italy \\ ${ }^{4}$ Protein Analysis Facility, Centre for Integrative Genomics, University of Lausanne, 1015 Lausanne, \\ Switzerland \\ ${ }^{5}$ Laboratory of Molecular and Cellular Biology, University of Neuchâtel, rue E-Argand 11, 2009 \\ Neuchâtel, Switzerland
}

In an acidic protein medium Aspergillus fumigatus secretes an aspartic endoprotease (Pep) as well as tripeptidyl-peptidases, a prolyl-peptidase and carboxypeptidases. In addition, LC-MS/MS revealed a novel glutamic protease, AfuGprA, homologous to Aspergillus niger aspergillopepsin II. The importance of AfuGprA in protein digestion was evaluated by deletion of its encoding gene in A. fumigatus wild-type D141 and in a pep $\Delta$ mutant. Either $A$. fumigatus Pep or AfuGprA was shown to be necessary for fungal growth in protein medium at low $\mathrm{pH}$. Exoproteolytic activity is therefore not sufficient for complete protein hydrolysis and fungal growth in a medium containing proteins as the sole nitrogen source. Pep and AfuGprA constitute a pair of endoproteases active at low $\mathrm{pH}$, in analogy to $A$. fumigatus alkaline protease (Alp) and metalloprotease I (Mep), where at least one of these enzymes is necessary for fungal growth in protein medium at neutral $\mathrm{pH}$. Heterologous expression of AfuGprA in Pichia pastoris showed that the enzyme is synthesized as a preproprotein and that the propeptide is removed through an autoproteolytic reaction at low $\mathrm{pH}$ to generate the mature protease. In contrast to A. niger aspergillopepsin II, AfuGprA is a single-chain protein and is structurally more similar to $\mathrm{G} 1$ proteases characterized in other non-Aspergillus fungi.

\section{INTRODUCTION}

Aspergillus fumigatus is an important opportunistic pathogen that is the main causative agent of invasive aspergillosis in neutropenic patients (Latgé, 1999). In composts, this fungus plays an important role in the decomposition of organic material and in recycling environmental carbon and nitrogen (Beffa et al., 1998). Like many other ascomycetes from the soil, A. fumigatus grows well in media containing protein as the sole nitrogen and carbon source and shows secreted proteolytic activity (Reichard et al., 1990; Monod et al., 1991; Sriranganadane et al., 2010). At neutral pH, the

Abbreviation: pNA, p-nitroaniline.

A supplementary figure showing the amino acid sequence alignment of A. fumigatus GprA (MER107323) with glutamic proteases from related taxa is available with the online version of this paper. fungus secretes two endoproteases, an alkaline protease (Alp) of the subtilisin family (Reichard et al., 1990; Monod et al., 1991) and a metalloprotease (Mep) of the fungalisin family (Monod et al., 1993a, b; Jaton-Ogay et al., 1994). In addition, A. fumigatus secretes leucine aminopeptidases (Laps), which are non-specific monoaminopeptidases, dipeptidyl-peptidases (DppIV and DppV) and a prolylpeptidase (AfuS28) as exopeptidases (Beauvais et al., 1997a, b; Monod et al., 2005; Sriranganadane et al., 2010). alp $\Delta$ mep $\Delta$ knockout mutants are unable to grow in a medium containing protein as the sole nitrogen source at neutral and high $\mathrm{pH}$ while single alp $\Delta$ and mep $\Delta$ mutants produced 30 and $70 \%$ of the proteolytic activity of the wild-type strain, respectively (Monod et al., 1993a, b; Jaton-Ogay et al., 1994).

In a protein medium at low $\mathrm{pH}, A$. fumigatus was found to secrete another set of proteases, including the aspartic 
protease Pep as an endoprotease (Reichard et al., 1995), tripeptidyl-peptidases (Tpp) of the sedolisin family (SedB to SedD) (Reichard et al., 2006), and AfuS28 and carboxypeptidases of the S10 family as exoproteases (Sriranganadane et al., 2010). In addition, we identified by MS an as yet uncharacterized putative glutamic endoprotease, AfuGprA (XP_748619, MER107323; encoding a gene at the locus AFUA_3G02970) (Monod et al., 2009; Sriranganadane et al., 2010), homologous to Aspergillus niger proteinase A or aspergillopepsin II (Takahashi, 2004) and to Scytalidium lignicolum scytalidopepsin B (Oda, 2004). A. niger aspergillopepsin II is not inhibited by pepstatin and was previously considered as a non-pepsin-type acid protease (Sasaki et al., 1995; Takahashi, 2004). The active site of scytalidopepsin B and aspergillopepsin II was identified with a catalytic dyad formed by residues Glu (E) and Gln (Q) (Fujinaga et al., 2004; Yabuki et al., 2004; Kataoka et al., 2005). This finding led to the establishment of a novel family of proteases called eqolisins (Fujinaga et al., 2004) or glutamic peptidases (Yabuki et al., 2004; Kataoka et al., 2005) separate from the aspartic proteases (see the MEROPS proteolytic enzyme database, http://merops.sanger.ac.uk).

A. fumigatus pep mutants were constructed in order to test the role of Pep in virulence (Reichard et al., 1997). However, no difference in pathogenicity was observed between the wild-type strain and pep mutants. Mortality curves were not statistically different, and histopathological studies of lungs from infected guinea pigs showed a similar extent of mycelium growth. In a control analysis, we found that A. fumigatus pep mutants grew well at low $\mathrm{pH}$ in a protein medium. Because this result suggested that other endoproteases could rescue a lack of Pep activity, here we characterized AfuGprA and investigated its function with respect to protein digestion. We show that AfuGprA and Pep constitute a pair of endoproteases in which one of them is necessary for fungal growth in a protein medium at low $\mathrm{pH}$.

\section{METHODS}

Fungal and bacterial strains. A. fumigatus D141 (NRRL 6585; US Department of Agriculture, Peoria, IL) and an isogenic pep $\Delta$ mutant (Reichard et al., 1997) were used in this study. All plasmid subcloning experiments were performed in Escherichia coli XL1 Blue. Pichia pastoris GS115 (Invitrogen) was used to produce recombinant AfuGprA.

Growth of A. fumigatus on solid media. A. fumigatus was grown on $2 \%(\mathrm{w} / \mathrm{v})$ malt agar medium (Oxoid) for production of spores, and on BSA agar medium and soy protein agar medium at $\mathrm{pH} 7.0$ or $\mathrm{pH}$ 4.0. BSA agar medium contained $1.2 \%(\mathrm{w} / \mathrm{v})$ yeast carbon base (YCB; Difco), $0.2 \%(\mathrm{w} / \mathrm{v})$ bovine serum albumin (Fraction V; Serva) and $1.5 \%(\mathrm{w} / \mathrm{v})$ agar. Nine hundred millilitres of a solution containing YCB and agarose was adjusted to $\mathrm{pH} 7.0$ or $\mathrm{pH} 4.0$ with $1 \mathrm{M} \mathrm{NaOH}$ or $1 \mathrm{M} \mathrm{HCl}$, respectively, and sterilized by autoclaving at $120{ }^{\circ} \mathrm{C}$. A BSA solution $(2.0 \%, \mathrm{w} / \mathrm{v}, 100 \mathrm{ml})$ with $\mathrm{pH}$ adjusted to 7.0 or 4.0 using $1 \mathrm{M} \mathrm{NaOH}$ or $1 \mathrm{M} \mathrm{HCl}$, respectively, was sterilized by filtration and added to cooled YCB-agarose and the mix was poured into Petri dishes. Soy protein agar medium contained $0.2 \%(w / v)$ soy protein (Supro 1711; Protein Technologies International) and $1.5 \%$ (w/v) agar. To test the growth of A. fumigatus at low $\mathrm{pH}$, soy protein was dissolved in $68 \mathrm{mM}$ citrate buffer $(\mathrm{pH} 4.0)$. Neutral and acidic soy protein media were sterilized by autoclaving at $120^{\circ} \mathrm{C}$ and poured into Petri dishes.

Liquid cultures of $\boldsymbol{A}$. fumigatus. To promote production of proteolytic activity at neutral $\mathrm{pH}$, A. fumigatus was grown in liquid medium containing protein as the sole nitrogen source $[1.2 \%(\mathrm{w} / \mathrm{v})$ BSA supplemented with $1.2 \%(\mathrm{w} / \mathrm{v})$ YCB or $0.2 \%(\mathrm{w} / \mathrm{v})$ soy protein]. The $\mathrm{pH}$ was adjusted to 7.0 with $1 \mathrm{M} \mathrm{NaOH}$ and was observed to increase slightly to $\mathrm{pH} 7.5$ during growth of the fungus. To test proteolytic activity at low $\mathrm{pH}$ in BSA liquid medium, $1.2 \%(\mathrm{w} / \mathrm{v}) \mathrm{BSA}$ supplemented with $1.2 \%$ (w/v) YCB was adjusted to $\mathrm{pH} 4.0$ with $1 \mathrm{M}$ $\mathrm{HCl}$. To test proteolytic activity at low $\mathrm{pH}$ in soy protein liquid medium, $0.2 \%(\mathrm{w} / \mathrm{v})$ soy protein was dissolved in $68 \mathrm{mM}$ citrate buffer ( $\mathrm{pH} 4.0$ ). One hundred millilitre flasks containing $50 \mathrm{ml}$ of medium were inoculated with approximately $10^{7}$ spores each and incubated for $96 \mathrm{~h}$ at $30{ }^{\circ} \mathrm{C}$ on an orbital shaker at 200 r.p.m. The $\mathrm{pH}$ of the medium was measured at $48 \mathrm{~h}, 72 \mathrm{~h}$ and the end of the incubation and was found to remain constant.

Gene replacement. AfugprA replacement was performed in A. fumigatus D141 and in the D141 peps mutant. The plasmid $\mathrm{p} \Delta \operatorname{gprA}$ was designed to function as a one-step gene replacement vector in transformation experiments and was constructed using pSK397 (Krappmann et al., 2005, 2006). The p $\Delta g p r A$ plasmid contained AfugprA replaced by the E. coli hygromycin B phosphotransferase gene $(h p h)$ under the control of a truncated gpd promoter and the trpC terminator of Aspergillus nidulans (Fig. 1). For plasmid construction, two approximately $1.2 \mathrm{~kb}$ fragments covering the $5^{\prime}$ - and $3^{\prime}$-flanking regions of AfugprA and small portions $(<100 \mathrm{bp})$ of this gene ( $\mathrm{fll}$ and Cflr, respectively) were PCRamplified using genomic A. fumigatus DNA as a template. P1/P2 and $\mathrm{P} 3 / \mathrm{P} 4$ primers, which contained additional restriction sites at the $5^{\prime}$ extremity for subsequent cloning steps (Table 1), were used to amplify $\mathrm{Nflr}$ and Cflr, respectively. Nflr and Cflr were subsequently digested with NotI/XmaI and PacI/HinDIII, respectively, and were inserted end-to-end into a modified pBluescript II SK $(+)$ (Stratagene) in which a PacI restriction site has been previously added in the multiple cloning site. The pBluescript construction generated contained, between unique NotI and PacI sites, Nflr and Cflr in the same orientation as in the A. fumigatus genome, and two different Sfil sites located between $\mathrm{Nflr}$ and $\mathrm{Cflr}$ for subsequent directive cloning (Kämper, 2004). Thereafter, the cassette containing the hygromycinresistance gene was excised with Sfil from pSK397 and cloned into the $S f i$ site of the pBluescript construction to generate $\mathrm{p} \Delta g p r A$.

Transformation of wild-type A. fumigatus D141 and the D141 peps mutant was accomplished according to a protocol that has been used for A. nidulans and A. fumigatus (Tilburn et al., 1983). Transformation of $10^{7}$ protoplasts with $5 \mu \mathrm{g} \mathrm{PacI}$ and Not I doubledigested p $\Delta g p r$ A typically yielded 100-200 hygromycin-resistant colonies. After overnight $h p h$ expression, the transformants were incubated on agar, based on GYE medium [1\%(w/v) glucose, $0.5 \%$ (w/v) yeast extract] containing $200 \mu \mathrm{g}$ hygromycin $\mathrm{ml}^{-1}$ (Sigma), and selected after 5 days of incubation at $20{ }^{\circ} \mathrm{C}$ followed by an overnight incubation at $42{ }^{\circ} \mathrm{C}$. Transformants initially identified as hygromycin-resistant were picked and subcultured again on agar containing hygromycin.

A. fumigatus gprAs mutants were identified by PCR of genomic DNA from various numbers of the hygromycin-resistant colonies as previously performed for selection of other protease- and transporter-deficient mutants (Reichard et al., 2006; Léchenne et al., 2007). Two pairs of specific $\Delta g p r A$ primers (P5/P6 and P7/P8, Table 1 ) were used to verify correct $g p r A$ replacement. In each primer pair, 
(a)
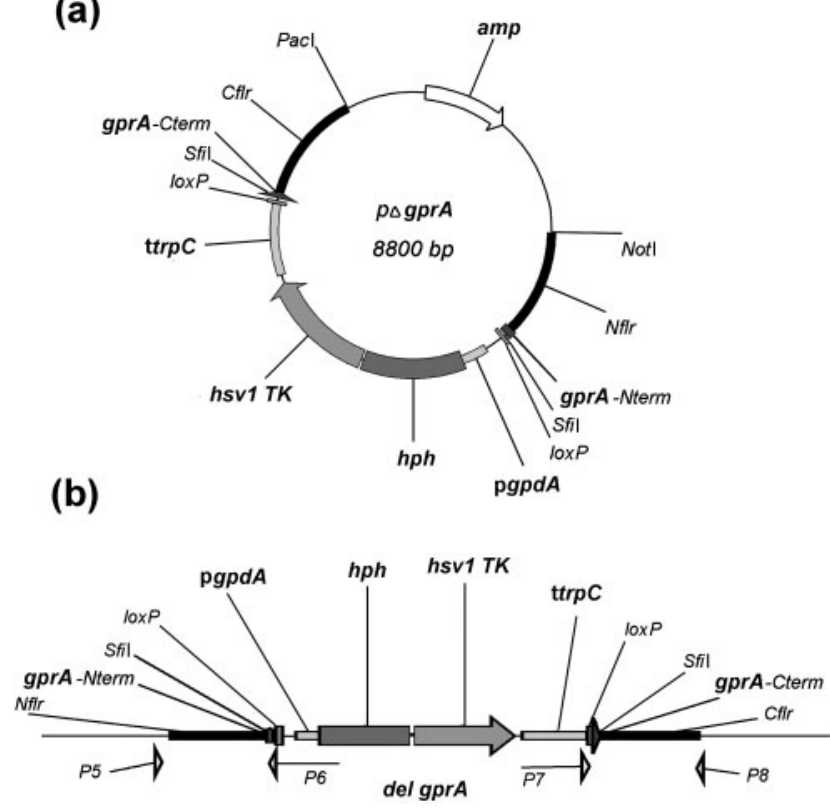

Fig. 1. (a) Map of $\mathrm{p} \Delta g p r A$ used for AfugprA replacement. (b) Genomic DNA of generated $A$. fumigatus gprA $\Delta$ mutants. The transforming DNA was excised from $\mathrm{p} \Delta g p r \mathrm{~A}$ via double digestion with Notl and Pacl. Screening for homologous integration was done by PCR with primer pairs P5/P6 and P7/P8 (see Methods). amp, ampicillin-resistance gene; gprA-Nterm, 3'-extremity of AfugprA; gprA-Cterm, 5'-extremity of AfugprA; hph, E. coli hygromycin B phosphotransferase gene; Nflr and Cflr, $1.2 \mathrm{~kb}$ fragments covering, respectively, the $\mathrm{N}$-terminal and $\mathrm{C}$-terminal flanking regions including small portions of AfugprA ( $<100 \mathrm{bp}$ ); hsv1 TK, thymidine kinase-encoding sequence from the herpes simplex virus type 1 ; lox $P$, specific recombinase binding sites of plasmid pSK397 (18-19); pgpdA, truncated $A$. nidulans gpd promoter; trpC, terminator region of the $A$. nidulans trp $C$ gene.

one primer hybridized with a part of the former transformation plasmid sequence and the other primer hybridized with genomic DNA near the desired homologous integration locus (Fig. 1). The predicted PCR product sizes were 1343 and 1350 bp with P5/P6 and P7/P8 primer pairs, respectively, if the transforming DNA was integrated at the homologous site. Two A. fumigatus D141 gprAs and two D141 pep $\Delta$ gprA $\Delta$ mutant strains were identified among 20 and 30 hygromycin-resistant colonies, respectively, which were tested for gene disruption.

Recombinant protease production. To construct $P$. pastoris strains producing GprA, DNA encoding N-terminal and C-terminal portions of the protein was amplified by PCR with a standard protocol using homologous sense and antisense primers (P9 and P10, Table 1) and $200 \mathrm{ng}$ A. fumigatus genomic DNA as no intron was predicted in the gene encoding AfuGprA (AfugprA). The PCR product was digested with $X h o I$ and $B g l I I$, for which a site was previously designed at the $5^{\prime}$ end of the primers and inserted into pKJ113 (Borg-von Zepelin et al., 1998) digested with $\mathrm{XhoI} / \mathrm{BamHI}$ to generate the expression plasmid pAfugprA. Transformation of $P$. pastoris GS115 with EcoRI-linearized plasmid DNA, selection of transformants and production of recombinant AfuGprA in methanol medium were performed as previously described (Borg-von Zepelin et al., 1998).
Purification of heterologously produced AfuGprA. AfuGprA was purified at $4{ }^{\circ} \mathrm{C}$ as follows. Secreted proteins from $250 \mathrm{ml} \mathrm{P}$. pastoris culture supernatant were concentrated by ultrafiltration to $6 \mathrm{ml}$ using a Centricon Plus-70 (10 kDa cut-off; Millipore). Thereafter, the concentrate was desalted with a PD10 column (Amersham Pharmacia) and applied to a DEAE-Sepharose column which had previously been equilibrated with $100 \mathrm{mM}$ Tris/HCl buffer ( $\mathrm{pH}$ 9.5). After washing the column with the same buffer, the recombinant protein was eluted with $100 \mathrm{mM}$ Tris/ $\mathrm{HCl}$ buffer ( $\mathrm{pH} 8$ ). Enzymic activity was tested with resorufin-labelled casein as a substrate (see below) and active fractions were pooled.

Protein gel electrophoresis. Extracts were analysed by SDS-PAGE with a separation gel of $12 \%$ polyacrylamide (Laemmli, 1970). The gels were stained either with $0.1 \%$ Coomassie brilliant blue R-250 (Bio-Rad) in ethanol/acetic acid/water $(40: 10: 50$, by vol.) or with silver nitrate according to Chevallet et al. (2006).

Protein identification by LC-MS/MS. Identification and confirmation of heterologously expressed proteins were obtained by a LC-MS/ MS analysis approach. Recombinant enzyme at $\mathrm{pH} 6.0$ or after 60 min incubation at room temperature in formic acid buffer ( $\mathrm{pH} 4.0$ ) was subjected to regular SDS-PAGE (12\%). Coomassieblue-stained bands were excised from the SDS-PAGE and in-gel digested with sequencing-grade chymotrypsin (Promega) as described by Shevchenko et al. (1996) and Wilm et al. (1996). Extracted peptides were analysed on a hybrid linear trap LTQ-Orbitrap mass spectrometer (Thermo Fisher) interfaced via a TriVersa Nanomate (Advion Biosciences) to an Agilent 1100 nano HPLC system (Agilent Technologies). Solvents used for the mobile phase were 95:5 (v/v) $\mathrm{H}_{2} \mathrm{O} /$ acetonitrile with $0.1 \%(\mathrm{v} / \mathrm{v})$ formic acid (solvent A) and 5:95 (v/v) $\mathrm{H}_{2} \mathrm{O} /$ acetonitrile with $0.1 \%(\mathrm{v} / \mathrm{v})$ formic acid (solvent $\mathrm{B}$ ). Peptides were loaded onto a trapping microcolumn (ZORBAX 300SB C18; $5 \mathrm{~mm} \times 300 \mu \mathrm{m}$ i.d., $5 \mu \mathrm{m}$; Agilent) in $97: 3(\mathrm{v} / \mathrm{v}) \mathrm{H}_{2} \mathrm{O} /$ acetonitrile plus $0.1 \%(\mathrm{v} / \mathrm{v})$ formic acid at a flow rate of $10 \mu \mathrm{l} \mathrm{min}{ }^{-1}$. After $5 \mathrm{~min}$, they were back-flush eluted and separated on a reversedphase nanocolumn (ZORBAX 300SB C18 column; $75 \mu \mathrm{m}$ i.d. $\times 15 \mathrm{~cm}, 3.5 \mu \mathrm{m}$; Agilent) at a flow rate of $300 \mathrm{nl} \mathrm{min}^{-1}$ with a gradient from 5 to $85 \%$ acetonitrile in $0.1 \%$ formic acid: $5 \mathrm{~min}$ at $0 \%$ solvent B, from 0 to $25 \% \mathrm{~B}$ in $35 \mathrm{~min}, 25$ to $50 \% \mathrm{~B}$ in $15 \mathrm{~min}, 50$ to $90 \%$ in $5 \mathrm{~min}, 90 \% \mathrm{~B}$ for $10 \mathrm{~min}, 90$ to $0 \%$ in $5 \mathrm{~min}$ and $15 \mathrm{~min}$ at $0 \%$ (total time: $90 \mathrm{~min}$ ).

For spraying, a 400-nozzle ESI Chip (Advion Biosciences) was used at a voltage of $1.65 \mathrm{kV}$, and the mass spectrometer capillary transfer temperature was set at $200{ }^{\circ} \mathrm{C}$. In data-dependent acquisition controlled by Xcalibur 2.0.7 software (ThermoFisher), the six most intense precursor ions detected in the full MS survey performed in the Orbitrap (range $350-1500 \mathrm{~m} / \mathrm{z}$, resolution 60000 at $\mathrm{m} / z$ 400) were selected and fragmented. MS/MS was triggered by a minimum signal threshold of 10000 counts, carried out at relative collision energy of $35 \%$ and with isolation width of $4.0 \mathrm{amu}$. Only precursors with a charge higher than 1 were selected for collision-induced dissociation (CID) fragmentation, and fragment ions were analysed in the LTQ linear trap. The $\mathrm{m} / \mathrm{z}$ of fragmented precursors was then dynamically excluded, with a tolerance of $0.01 \mathrm{amu}$, from any selection for $120 \mathrm{~s}$.

From raw files, MS/MS spectra were exported as $m g f$ (Mascot Generic File, text format) files using the extract_msn.exe script (Thermo Fisher) with the following settings: peptide mass range, 500-5000; minimum total ion intensity threshold, 500; minimum number of fragment ions, 15; minimum signal-to-noise ratio needed for a peak to be written, 3 .

Samples were analysed using Mascot 2.2 (Matrix Science). Mascot was set up to search a custom-built database containing the sequences of the glutamic protease AfuGprA and of contaminants (enzymes, keratins). Semi-specific cleavage at F, L, W and Y (not before P) was 
Table 1. Primers used in this study

Primers P1-P4 were used to construct Nflr and Cflr fragments. Primers P5-P8 were used for screening A. fumigatus gprA $\Delta$ mutants. For location of those primers in the A. fumigatus gprAs mutant, see Fig. 1. Primers P9 and P10 were used for AfugprA gene expression in P. pastoris.

\begin{tabular}{|c|c|c|c|}
\hline Primer & Sequence $\left(5^{\prime}-3^{\prime}\right)^{*}$ & Location & $\begin{array}{l}\text { PCR product size with } \\
\text { cloning sites }\end{array}$ \\
\hline P1 & TATATGCGGCCGCCCATATTCGTCTGGCTCACC & Upstream sequence in AfugprA & $1192 \mathrm{bp}$ (Nflr fragment) \\
\hline P2 & $\begin{array}{l}\text { ATATTA } C C C G G G G G C C T G A G T G G C C C T T G A C G C \\
\text { TGTTCTGTGAGG }\end{array}$ & Complement of AfugprA, $\mathrm{N}$-terminal region & NotI-SfiI-XmaI \\
\hline P3 & $\begin{array}{l}\text { ATATTAAAGCTTGGCCATCTAGGCCAGCGGCAA } \\
\text { GGTTTTGACTT }\end{array}$ & Downstream sequence in AfugprA & 1219 bp (Cflr fragment) \\
\hline $\mathrm{P} 4$ & TATCGTTAATTAAATGAACCGCCTTCAGAGCTA & Complement of AfugprA, C-terminal region & HindIII-SfiI-PacI \\
\hline P5 & GGGCTGAAGGAAGAATACCC & Upstream sequence in AfugprA & $1343 \mathrm{bp}$ \\
\hline P6 & AGGTGATATCGGCCTGAGTG & pSK397 & \\
\hline P7 & CGTTTACCCAGAATGCACAG & Downstream sequence in AfugprA & $1350 \mathrm{bp}$ \\
\hline P8 & GCACAGTGATATCGGACGAC & pSK397 & \\
\hline P9 & GTTCTCGAGCCATCGCTGCTCCCCTCACA & AfugprA & $782 \mathrm{bp}$ \\
\hline $\mathrm{P} 10$ & GTTGGATCCTTAGACATACTTAACAGTGAC & Complement of AfugprA & XhoI-BamHI \\
\hline
\end{tabular}

${ }^{*}$ Nucleotides shown in italics and bold represent cloning sites.

used as the enzyme definition, with a maximum of two missed cleavages allowed. Mascot was searched with a fragment ion mass tolerance of $0.50 \mathrm{Da}$ and a parent ion tolerance of 10 p.p.m. The iodoacetamide derivative of cysteine was specified in Mascot as a fixed modification. Deamidation of asparagine and glutamine, oxidation of methionine and protein $\mathrm{N}$-acetylation were specified as variable modifications.

Proteolytic activities. Endoproteolytic activity was measured with $0.02 \%(w / v)$ resorufin-labelled casein as a substrate (Roche Diagnostics) at different $\mathrm{pHs}$ in sodium citrate buffer $(50 \mathrm{mM}$ final concentration; $\mathrm{pH} 2.0-7.0$ ) in a total volume of $0.5 \mathrm{ml}$ (Borg-von Zepelin et al., 1998; Reichard et al., 2006). After incubation at $37{ }^{\circ} \mathrm{C}$, the undigested substrate of the enzyme/substrate mix was precipitated by trichloroacetic acid $(4 \%, \mathrm{v} / \mathrm{v}$, final concentration) and separated from the supernatant by centrifugation. Five hundred microlitres of Tris/ $\mathrm{HCl}$ buffer (500 $\mathrm{mM}$; pH 9.4) was added to the collected supernatant (neutralization step) and the $A_{574}$ of the mixture ( $1 \mathrm{ml}$ ) was measured. For practical purposes, $1 \mathrm{mU}$ activity was defined as producing an increase in absorbance of $0.001 \mathrm{~min}^{-1}$ in a proteolytic assay $(1 \mathrm{ml})$ at optimal $\mathrm{pH}$ for activity. Activities were measured in culture supernatant without and with the presence of the aspartic protease inhibitor pepstatin at a concentration of $10 \mu \mathrm{g} \mathrm{ml}^{-1}$.

Tripeptidyl-peptidase and AfuS28 activities were measured as previously described, using Phe-Pro-Ala-pNA and Ala-Ala-Ala-pNA (Bachem), respectively, as substrates (Reichard et al., 1997; Sriranganadane et al., 2010). The reaction mixture contained $80 \mu \mathrm{l}$ culture supernatant, $5 \mathrm{mM}$ substrate and $50 \mathrm{mM}$ citrate buffer $(\mathrm{pH} 4)$ in a total volume of $100 \mu \mathrm{l}$. After incubation at $37^{\circ} \mathrm{C}$ for $0.5-$ $4 \mathrm{~h}$, the reaction was terminated by adding $5 \mu \mathrm{l}$ glacial acetic acid. The $p$-nitroaniline (pNA) released was measured by spectrometry as a change in $A_{405}$. A control with a blank substrate and blank culture broth was carried out in parallel. Enzyme activities were expressed in $\mathrm{mU}$ (nmol released $p \mathrm{NA} \min ^{-1}$ ).

Phylogenetic analyses. Amino acid sequences of a selection of putative glutamic proteases from Aspergillus spp., and previously characterized proteases from the ascomycetes Talaromyces emersonii, Botryotinia fuckeliana, Sclerotinia sclerotium, Scytalidium lignicolum and Cryphonectria parasitica (MEROPS G01 family) were aligned using Clustal $\mathrm{W}$ as implemented in BioEdit Sequence Alignment Editor software (Hall, 1999). Phylogenetic analyses were performed using the following reconstruction methods and parameters: PhyML (Guindon \& Gascuel, 2003) with SH-like approximate likelihood ratio test, four substitution rate categories, and estimation of gamma distribution parameter and proportion of invariable sites; BIONJ (Gascuel, 1997), using a Dayhoff PAM substitution matrix; TNT (Goloboff et al., 2008) with sectorial search and tree fusing options; and MrBayes (Ronquist \& Huelsenbeck, 2003) with GTR likelihood model and 100000 Markov chain Monte Carlo generations. Phylogenetic trees were edited by using Dendroscope (Huson et al., 2007).

\section{RESULTS}

\section{Importance of AfuGprA in extracellular protein digestion}

To evaluate the importance of AfuGprA in protein digestion at low $\mathrm{pH}$, AfugprA was replaced in A. fumigatus wild-type D141 and pep $\Delta$ mutant as described in Methods. A. fumigatus wild-type D141, and pep $\Delta$, gprA $\Delta$ and pep $\Delta$ gprA $\Delta$ mutants were first tested for their ability to grow using protein solid and liquid media at neutral and low $\mathrm{pH}$ (Fig. 2). Two different protein sources, BSA and soy protein, which is a heterogeneous peptide mixture (Fig. 3c), were used.

No phenotypic difference was observed between wild-type and mutant strains at neutral $\mathrm{pH}$. At low $\mathrm{pH}$, A. fumigatus gprA $\Delta$ could not be differentiated from the wild-type D141 strain using the two protein sources. In contrast, A. fumigatus pep $\Delta$ gprA $\Delta$ was unable to grow on BSA agar medium at low $\mathrm{pH}$ whereas $A$. fumigatus pep $\Delta$ showed only slightly reduced colony size (Fig. 2). The four A. fumigatus strains showed comparable growth rate on soy protein agar medium at low pH (Fig. 2). 


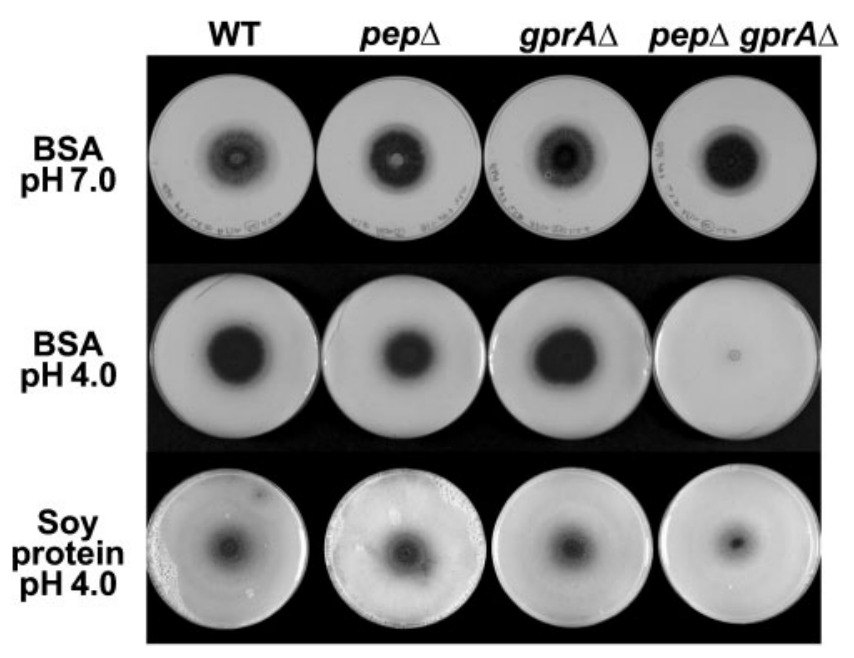

Fig. 2. Growth of $A$. fumigatus wild-type D141 (WT), and pep $\Delta$, gprA $\Delta$ and pep $\Delta$ gprA $\Delta$ mutants on BSA and soy protein agar medium.

Similar results were obtained with liquid cultures at low $\mathrm{pH}$ : A. fumigatus wild-type D141, and peps and gprA $\Delta$ mutants grew well in BSA and soy protein liquid medium; and only residual growth of A. fumigatus pep $\Delta$ gprAs without clarification of the medium was observed with BSA liquid medium, while the fungus grew well with soy protein (data not shown). After $96 \mathrm{~h}$ growth in the latter medium, the secreted activity of the $g p r A \Delta$ and pep $\Delta$ mutants was approximately 70 and $20 \%$ of that of A. fumigatus wildtype D141, respectively (Fig. 4). In the presence of the aspartic protease inhibitor pepstatin, activity of the $g \operatorname{pr} A \Delta$ mutant was totally inhibited and activity of the wild-type strain was reduced to the rate of that of the pep $\Delta$ mutant. A. fumigatus pep $\Delta$ gprA $\Delta$ soy protein culture supernatant was completely devoid of extracellular endoproteolytic activity. Comparable tripeptidyl-peptidase and AfuS28 activities were found to be secreted at low $\mathrm{pH}$ by A. fumigatus wild-type D141, and pep $\Delta, g p r A \Delta$ and pep $\Delta$ gprA $\Delta$ mutants in soy protein medium, as well as by A. fumigatus D141, pep $\Delta$ and gprAs in BSA medium (mean $\pm \mathrm{SD} 7.6 \pm 1.0$ and $0.03 \pm 0.01 \mathrm{mU}$, respectively). The production of these exoproteases was apparently not changed by deleting pep and gprA.

\section{Characterization of AfuGprA}

SDS-PAGE results of culture supernatants of $A$. fumigatus wild-type D141, pep $\Delta, g p r A \Delta$ and $p e p \Delta g p r A \Delta$ are shown in Fig. 3. The absence of a $36 \mathrm{kDa}$ band in A. fumigatus pep $\Delta$ and a $26 \mathrm{kDa}$ band in A. fumigatus gprA $\Delta$ mutants identified Pep and GprA, respectively. AfuGprA was characterized as a recombinant enzyme using $P$. pastoris as an expression system. A yield of $25 \mu \mathrm{g} \mathrm{ml}^{-1}$ recombinant protein in culture supernatant was obtained. Recombinant AfuGprA in $P$. pastoris culture supernatant showed an apparent molecular mass of $30 \mathrm{kDa}$, which is higher than that of AfuGprA secreted by A. fumigatus at $\mathrm{pH} 4$ (Fig. 5, lane 1). However, after pre-incubation at $\mathrm{pH} 4.0$, recombinant AfuGprA showed the same molecular mass as native AfuGprA (compare Fig. 5, lane 2, and Fig. 3). These results suggest that AfuGprA, like other G1 proteases, is made as a preproprotein and that the propeptide was cleaved and removed to generate the active enzyme through an autoproteolytic reaction.

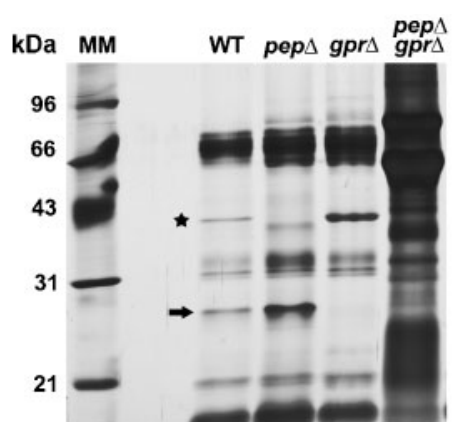

(a)

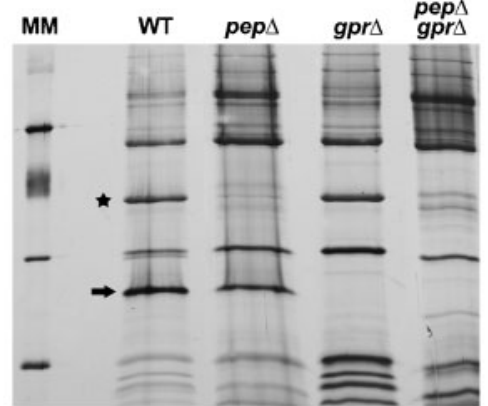

(b)

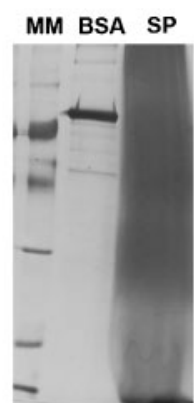

(c)

Fig. 3. $(a, b)$ Identification of Pep and GprA in culture supernatant of $A$. fumigatus wild-type D141 (WT) and pep $\Delta$, gprA $\Delta$ and pep $\Delta$ gprA $\Delta$ mutant strains growing in BSA (a) and soy protein (b) acidic liquid medium ( $\mathrm{pH} 4.0$ ). Flasks (200 ml) containing $50 \mathrm{ml}$ medium were inoculated with approximately $10^{7}$ spores and incubated for $96 \mathrm{~h}$ at $30{ }^{\circ} \mathrm{C}$ on an orbital shaker at 200 r.p.m. Supernatant extracts were concentrated by ultrafiltration and acidic precipitation as previously described (Sriranganadane et al., 2010). The precipitate from $2.0 \mathrm{ml}$ culture supernatant was resuspended in $10 \mu$ I SDS-PAGE buffer, and subsequently loaded on SDS-12\% polyacrylamide gels. (c) SDS-12 \% polyacrylamide gels with $1 \mu$ non-digested BSA and soy protein liquid medium $(1 \mu \mathrm{g}$ protein). The gels were stained with silver nitrate according to Chevallet et al. (2006). The bands corresponding to Pep and GprA are marked with an asterisk and an arrow, respectively, in panels (a) and (b). MM, molecular mass markers. 


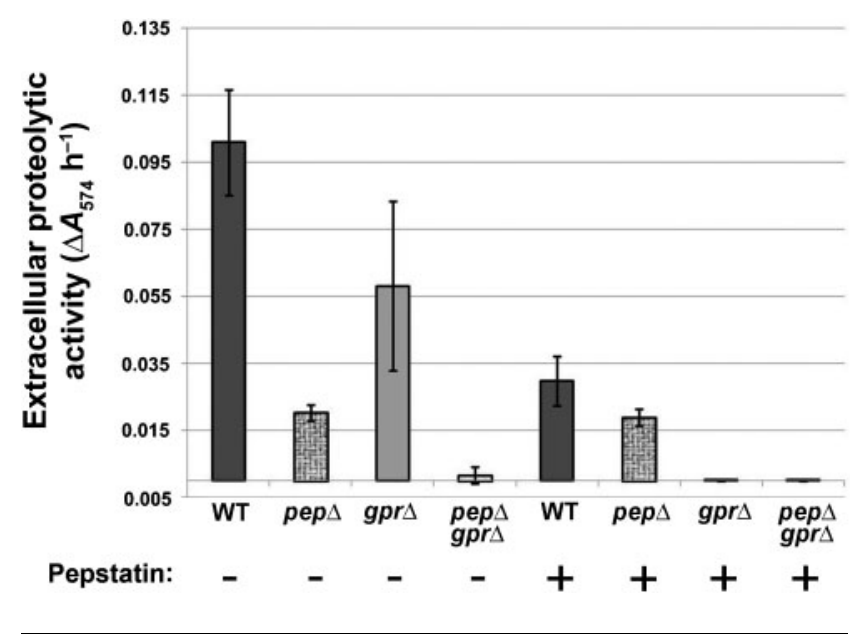

Fig. 4. Secreted endoproteolytic activity of $A$. fumigatus wild-type $\mathrm{D} 141$, and $p e p \Delta, g p r A \Delta$ and $p e p \Delta$ gprA $\Delta$ mutants in soy protein medium ( $\mathrm{pH} 4.0)$ after $96 \mathrm{~h}$ growth at $30{ }^{\circ} \mathrm{C}$. Activities from $50 \mu \mathrm{l}$ culture supernatant were measured as described in Methods using resorufin-labelled casein as a substrate in the absence and in the presence of pepstatin $\left(10 \mu \mathrm{g} \mathrm{ml}^{-1}\right)$. Means $\pm \mathrm{SD}$ of values recorded in three independent experiments are indicated.

The deduced amino acid sequence of the AfugprA open reading frame shows a 16-18 amino acid hydrophobic signal peptide with a putative signal peptidase cleavage site Ala-IleAla in accordance with von Heijne's rule (von Heijne, 1986)

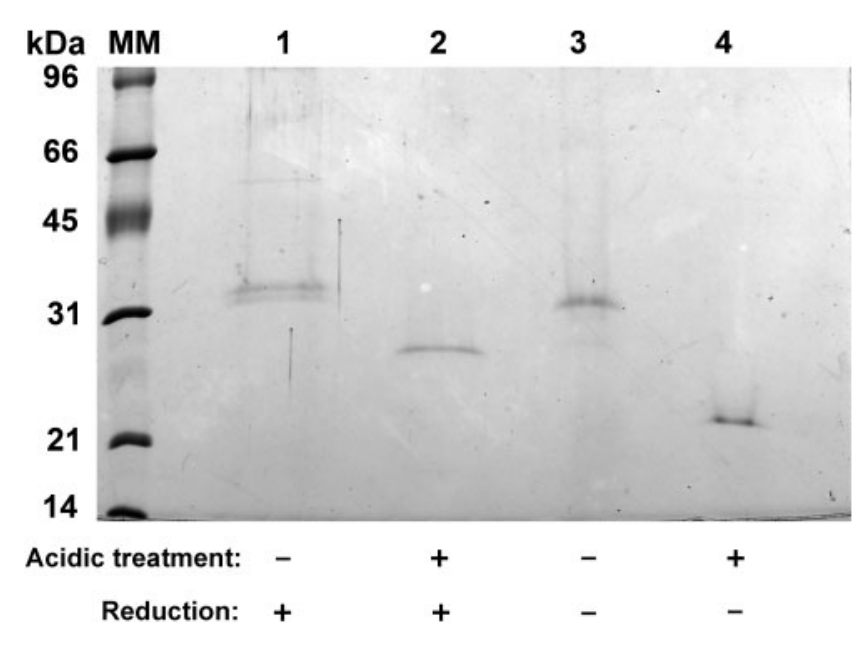

Fig. 5. SDS-PAGE ( $9 \%$ gel) of recombinant AfuGprA secreted by $P$. pastoris. The gel was stained with Coomassie blue. Approximately $0.3 \mu \mathrm{g}$ recombinant reduced (lanes 1 and 2) and non-reduced (lanes 3 and 4) AfuGprA was loaded on the gel. AfuGprA reduction was performed after heat treatment in SDSPAGE buffer containing $2 \%(\mathrm{v} / \mathrm{v}) \beta$-mercaptoethanol. In lanes 2 and 4, recombinant AfuGprA was incubated at $\mathrm{pH} 4.0$ for $30 \mathrm{~min}$ at $30{ }^{\circ} \mathrm{C}$. The double band observed for native proGprA attests to two different signal peptidase cleavage sites (see Fig. 6). MM, molecular mass markers. (see Supplementary Fig. S1, available with the online version of this paper). The AfuGprA proprotein generated after signal peptidase cleavage comprised 252 amino acids, with a theoretical mass of $26.34 \mathrm{kDa}$. Therefore, the molecular mass of reduced proAfuGprA was overestimated in SDS-PAGE gels as it migrates as a $30 \mathrm{kDa}$ protein. Of particular note, a similar observation was made with Botrytis cinerea secreted G1 protease (Rolland et al., 2009). Nonreduced proAfuGprA and AfuGprA showed a higher electrophoretic mobility than reduced proAfuGprA and AfuGprA, respectively, after a 2 min heating treatment in SDS-PAGE buffer (Fig. 5, lanes 3 and 4).

Identification of the heterologously produced AfuGprA was also confirmed using electrospray ionization (ESI)-LCMS/MS. Chymotryptic digestion was performed in gel slices containing the $30 \mathrm{kDa}$ protein secreted by $P$. pastoris at neutral $\mathrm{pH}$, and the $26 \mathrm{kDa}$ protein obtained after treatment at $\mathrm{pH} 4.0$ (Fig. 5). MS analysis allowed the detection of different AfuGprA peptides matching with residues $42-269$ in the $30 \mathrm{kDa}$ secreted protein. Peptides matching with residues $61-269$ but not with residues 1-61 were detected in the $26 \mathrm{kDa}$ protein (Fig. 6). These results confirmed that active AfuGprA was generated after cleavage of a prosequence at low $\mathrm{pH}$ at residue 61 or a few residues before as suggested from the alignment with other glutamic proteases. Gln and Glu residues of the catalytic dyad of AfuGprA were identified at positions 120 and 206, respectively (Fig. S1).

Characterization assays of the recombinant AfuGprA were done after purification by ion-exchange chromatography. The enzyme was found to be active between $\mathrm{pH} 2.0$ and 6.0 with an optimum activity at $\mathrm{pH} 5.0$ on resorufin-labelled casein. The specific activity of AfuGprA was $2.2 \mathrm{mU}$ ( $\mu \mathrm{g}$ protein $^{-1}$ at $\mathrm{pH}$ 5.0. The enzyme was not inhibited by pepstatin (aspartic protease inhibitor), E64 or iodoacetamide (cysteine protease inhibitors), EDTA or phosphoramidon

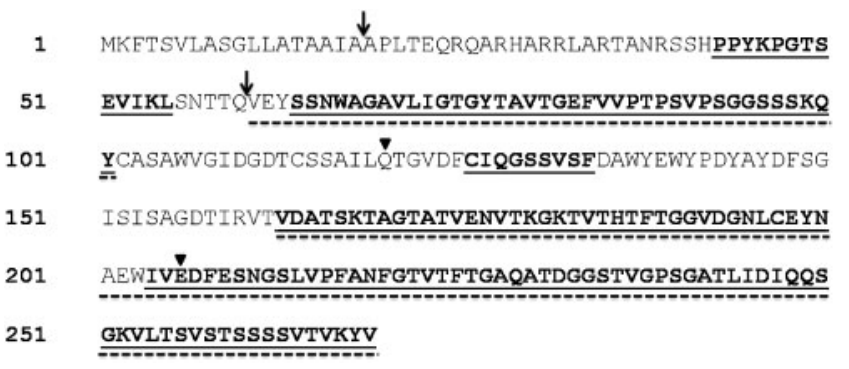

Fig. 6. Amino acid sequence of PreproAfuGprA. MS-detected peptides generated by chymotryptic digestion of proAfuGprA secreted by $P$. pastoris and mature AfuGprA after incubation at low $\mathrm{pH}$ are underlined with full and dashed lines, respectively. Vertical arrows indicate two potential signal peptidase cleavage sites at amino acid positions 16 and 18, and the cleavage site in the proprotein generating the mature enzyme. Active-site residues Gln and Glu forming the catalytic diad are indicated by solid triangles. 


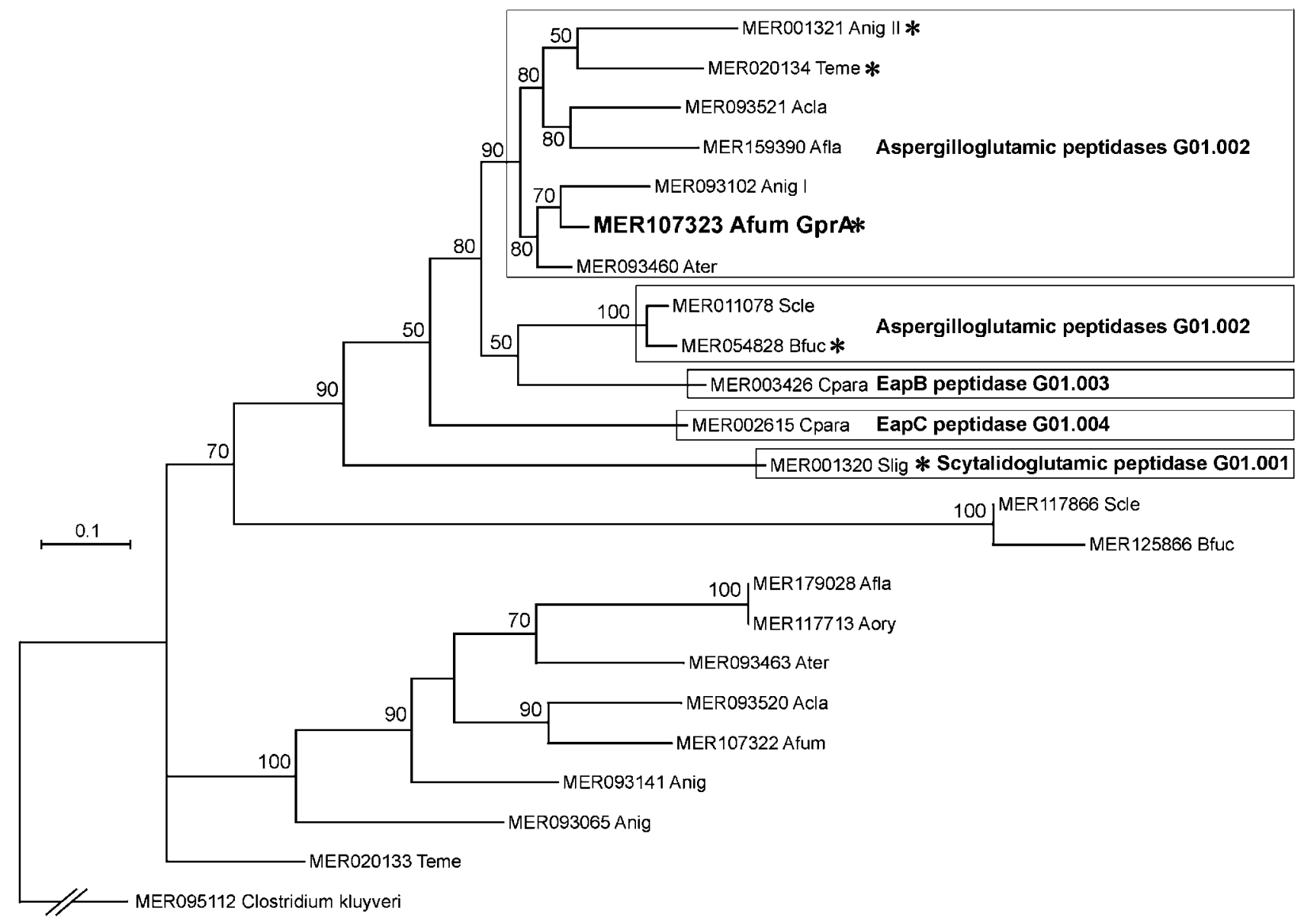

Fig. 7. Phylogenetic position of $A$. fumigatus GprA with respect to other fungal glutamic proteases obtained by a maximumlikelihood analysis using PhyML. Asterisks indicate proteases that have been characterized biochemically. A glutamic protease from Clostridium kluyveri (Firmicutes, Bacteria) was used a potential outgroup. MEROPS identifiers and names of type proteases are indicated. Numbers at nodes are approximate likelihood-ratio test values of branch support. The scale bar indicates the number of expected changes per site. Abbreviations: Afum, Aspergillus fumigatus; Anig, Aspergillus niger; Acla, Aspergillus clavatus; Ater, Aspergillus terreus; Afla, Aspergillus flavus; Teme, Talaromyces emersonii; Scle, Sclerotinia sclerotium; Bfuc, Botryotinia fuckelania; Cpara, Cryphonectria parasitica; Slig, Scytalidium lignicolum.

(metalloprotease inhibitors), or PMSF, antipain, chymostatin, leupeptin or aprotinin (serine protease inhibitors). Being resistant to class-specific inhibitors of aspartic, cysteine, metallo- and serine proteases, AfuGprA behaves like other previously described glutamic proteases (Oda, 2004; Takahashi, 2004; O’Donoghue et al., 2008).

\section{DISCUSSION}

Two endoproteases, Pep and GprA, are secreted by A. fumigatus in an acidic protein medium. We have shown that either A. fumigatus Pep or GprA was necessary for fungal growth in BSA medium at low pH. AfuGprA deficiency alone did not affect $A$. fumigatus growth while a slight growth rate reduction of the single pep $\Delta$ mutant was detected. These results apparently contrast with those obtained with $T$. emersonii, growth of which is strongly inhibited with glutamic peptidase antagonists (O'Donoghue et al., 2008). However, the G1 inhibitors tested could affect functions in the fungus other than G1 protease activity.

During the process of protein digestion the main function of the endoproteases is to produce large numbers of freeend peptides on which exopeptidases may act. Synergism of non-specific amino peptidases (Lap at neutral $\mathrm{pH}$, Seds at low $\mathrm{pH}$ ) and prolyl peptidases (DppIV or AfuS28 at neutral $\mathrm{pH}$, AfuS28 at low $\mathrm{pH}$ ) allows sequential degradation of large peptides previously generated by endoproteolysis into amino acids, and di- and tripeptides (Byun et al., 2001; Sriranganadane et al., 2010). Large peptide cleavage by further exoproteolytic activity is also essential for fungi using protein as sole nitrogen and carbon source as only amino acids and short peptides can be assimilated by transporters. When using soy protein as source, which is a heterogeneous peptide mixture, exoproteolytic activity alone was sufficient for fungal growth. 
Two major proteases, aspergillopepsin A (Pep) and aspergillopepsin B (aspergillopepsin II), were found in the culture filtrate of $A$. niger (Koaze et al., 1964). An A. niger mutant deficient in the secretion of both proteases was generated by UV mutagenesis (Mattern et al., 1992). This strain, in which a mutation is located in a gene encoding a regulator of extracellular protease genes called PrtT (Punt et al., 2008), has only 1-2\% extracellular protease activity at $\mathrm{pH}$ 5.3. In contrast, $A$. fumigatus pep $\Delta$ gprA $\Delta$ produces substantial proteolytic activity and grows well in a protein medium at this $\mathrm{pH}$ because of the secretion of Alp and Mep (data not shown). Both enzymes are still active at pH 5.3 (Monod et al., 1991, 1993b). The A. fumigatus genome contains an orthologue of $A$. niger PRTT, which was shown to control the expression of multiple secreted proteases such as Alp, Mep, Pep, SedB and DppIV (Sharon et al., 2009; Bergmann et al., 2009). However, functional PrtT dependence of A. fumigatus secreted proteases was investigated at neutral $\mathrm{pH}$ only, and the role of PrtT in protein gene expression was not addressed at low $\mathrm{pH}$. Virulence studies were not within the scope of the present investigations, but $A$. fumigatus prtT deletion mutants were shown to be not attenuated in virulence in immunosuppressed mice (Sharon et al., 2009; Bergmann et al., 2009).

Being a single-chain protein, mature AfuGprA resembles other previously characterized fungal glutamic proteases from $T$. emersonii, S. lignicolum and $B$. fuckeliana. However, it differs from A. niger aspergillopepsin II, which is a two-chain protein composed of a 39-residue light chain and a 173-residue heavy chain. Both chains of aspergillopepsin II are non-covalently bound and originate from a single precursor of 282 amino acids which contains a 14 amino acid intervening peptide (Fig. S1) that is absent in the AfuGprA preproprotein. The heterodimer results from autoproteolytic cleavage at the level of the intervening peptide as for heterodimer yapsins, where an autocleavage occurs within an internal loop of the polypeptide chain (Gagnon-Arsenault et al., 2006). In fact, none of the currently available sequences of fungal glutamic proteases possesses the intervening peptide found in A. niger aspergillopepsin II. This two-chain glutamic protease seems therefore to be an exception.

Sequence searches in the GenBank and MEROPS databases, sequence alignments and phylogenetic analyses indicated that putative orthologues of AfuGprA occur in all Aspergillus species (Fig. 7). Two G1 proteases are found in the A. fumigatus genome, GprA (MER107323) and a second G1 protease (MER107322) which was not detected in the present study. AfuGprA belongs to a robust clade including all aspergilloglutamic peptidases (MEROPS identifier G01.002) from Aspergillus spp. and T. emersonii. AfuGprA is related most closely to MER093102 of A. niger ( $76 \%$ identity, $89 \%$ similarity), a putative protease. Characterized aspergilloglutamic peptidases from Sclerotinia sclerotium and $B$. fuckeliana, as well as glutamic proteases from C. parasitica and Scytalidium lignicolum (G01.001, G01.003 and G01.004), branch basally with respect to Aspergillus G01.002 peptidases. It may be noted that most fungal species possess at least two paralogous genes encoding glutamic proteases. Each species typically harbours an aspergilloglutamic-like protease belonging to the G01.001-G01.004 clades and a glutamic protease designated 'unassigned' in the MEROPS database and branching at the base of the tree. None of these unassigned glutamic proteases has been characterized experimentally to date. The relatively high amino acid sequence divergence between the glutamic proteases found in a given genome (only $37 \%$ identity between A. fumigatus GprA and A. fumigatus unassigned protease MER107322) together with the scattered distribution of sequences in the phylogenetic tree suggests that these paralogues arose by ancient gene duplication.

In conclusion, we have shown that secreted glutamic protease rescues aspartic protease Pep deficiency in A. fumigatus and that exoproteolytic activity is not sufficient for complete protein hydrolysis and fungal growth in an acidic protein medium. Pep and GprA constitute a pair of acidic endoproteases analogous to the A. fumigatus Alp/Mep pair (Jaton-Ogay et al., 1994) at neutral $\mathrm{pH}$, in which expression of either one of them was shown to be sufficient for fungal growth in a protein medium.

\section{ACKNOWLEDGEMENTS}

We thank Sven Krappmann for providing pSK397 plasmid, Massimo Lurati for critical review of the manuscript and assistance with the English, and Olympia Bontems for helpful discussion and technical assistance. This work was supported by the Swiss National Foundation for Scientific Research, grant 320030-1179641.

\section{REFERENCES}

Beauvais, A., Monod, M., Debeaupuis, J. P., Diaquin, M., Kobayashi, H. \& Latgé, J. P. (1997a). Biochemical and antigenic characterization of a new dipeptidyl-peptidase isolated from Aspergillus fumigatus. J Biol Chem 272, 6238-6244.

Beauvais, A., Monod, M., Wyniger, J., Debeaupuis, J. P., Grouzmann, E., Brakch, N., Svab, J., Hovanessian, A. G. \& Latgé, J. P. (1997b). Dipeptidyl-peptidase IV secreted by Aspergillus fumigatus, a fungus pathogenic to humans. Infect Immun 65, 3042-3047.

Beffa, T., Staib, F., Lott Fischer, J., Lyon, P. F., Gumowski, P., Marfenina, O. E., Dunoyer-Geindre, S., Georgen, F., Roch-Susuki, R. \& other authors (1998). Mycological control and surveillance of biological waste and compost. Med Mycol 36 (Suppl. 1), 137-145.

Bergmann, A., Hartmann, T., Cairns, T., Bignell, E. M. \& Krappmann, S. (2009). A regulator of Aspergillus fumigatus extracellular proteolytic activity is dispensable for virulence. Infect Immun 77, 4041-4050.

Borg-von Zepelin, M., Beggah, S., Boggian, K., Sanglard, D. \& Monod, M. (1998). The expression of the secreted aspartyl proteinases Sap4 to Sap6 from Candida albicans in murine macrophages. Mol Microbiol 28, 543-554.

Byun, T., Kofod, L. \& Blinkovsky, A. (2001). Synergistic action of an $\mathrm{X}$-prolyl dipeptidyl aminopeptidase and a non-specific aminopeptidase in protein hydrolysis. J Agric Food Chem 49, 2061-2063. 
Chevallet, M., Luche, S. \& Rabilloud, T. (2006). Silver staining of proteins in polyacrylamide gels. Nat Protoc 1, 1852-1858.

Fujinaga, M., Cherney, M. M., Oyama, H., Oda, K. \& James, M. N. (2004). The molecular structure and catalytic mechanism of a novel carboxyl peptidase from Scytalidium lignicolum. Proc Natl Acad Sci U S A 101, 3364-3369.

Gagnon-Arsenault, I., Tremblay, J. \& Bourbonnais, Y. (2006). Fungal yapsins and cell wall: a unique family of aspartic peptidases for a distinctive cellular function. FEM Yeast Res 6, 966978.

Gascuel, O. (1997). BIONJ: an improved version of the NJ algorithm based on a simple model of sequence data. Mol Biol Evol 14, 685695.

Goloboff, P., Farris, J. \& Nixon, K. (2008). TNT: a free program for phylogenetic analysis. Cladistics 24, 774-786.

Guindon, S. \& Gascuel, O. (2003). A simple, fast, and accurate algorithm to estimate large phylogenies by maximum likelihood. Syst Biol 52, 696-704.

Hall, T. A. (1999). BioEdit: a user-friendly biological sequence alignment editor and analysis program for Windows 95/98/NT. Nucleic Acids Symp Ser 41, 95-98.

Huson, D. H., Richter, D. C., Rausch, C., Dezulian, T., Franz, M. \& Rupp, R. (2007). Dendroscope: an interactive viewer for large phylogenetic trees. BMC Bioinformatics 8, 460.

Jaton-Ogay, K., Paris, S., Huerre, M., Quadroni, M., Falchetto, R., Togni, G., Latgé, J. P. \& Monod, M. (1994). Cloning and disruption of the gene encoding an extracellular metalloprotease of Aspergillus fumigatus. Mol Microbiol 14, 917-928.

Kämper, J. (2004). A PCR-based system for highly efficient generation of gene replacement mutants in Ustilago maydis. Mol Genet Genomics 271, 103-110.

Kataoka, Y., Takada, K., Oyama, H., Tsunemi, M., James, M. N. \& Oda, K. (2005). Catalytic residues and substrate specificity of scytalidoglutamic peptidase, the first member of the eqolisin in family (G1) of peptidases. FEBS Lett 579, 2991-2994.

Koaze, Y., Goi, H., Ezawa, K., Yamada, Y. \& Hara, T. (1964). Fungal proteolytic enzymes. Part I. Isolation of two kinds of acid proteases excreted by Aspergillus niger var macrosporus. Agric Biol Chem 28, 216-223.

Krappmann, S., Bayram, O. \& Braus, G. H. (2005). Deletion and allelic exchange of the Aspergillus fumigatus veA locus via a novel recyclable marker module. Eukaryot Cell 4, 1298-1307.

Krappmann, S., Sasse, C. \& Braus, G. H. (2006). Gene targeting in Aspergillus fumigatus by homologous recombination is facilitated in a nonhomologous end-joining-deficient genetic background. Eukaryot Cell 5, 212-215.

Laemmli, U. K. (1970). Cleavage of structural proteins during the assembly of the head of bacteriophage T4. Nature 227, 680685.

Latgé, J. P. (1999). Aspergillus fumigatus and aspergillosis. Clin Microbiol Rev 12, 310-350.

Léchenne, B., Reichard, U., Zaugg, C., Fratti, M., Kunert, J., Boulat, O. \& Monod, M. (2007). Sulphite efflux pumps in Aspergillus fumigatus and dermatophytes. Microbiology 153, 905-913.

Mattern, I. E., van Noort, J. M., van den Berg, P., Archer, D. B., Roberts, I. N. \& van den Hondel, C. A. (1992). Isolation and characterization of mutants of Aspergillus niger deficient in extracellular proteases. Mol Gen Genet 234, 332-336.

Monod, M., Togni, G., Rahalison, L. \& Frenk, E. (1991). Isolation and characterisation of an extracellular alkaline protease of Aspergillus fumigatus. J Med Microbiol 35, 23-28.
Monod, M., Paris, S., Sarfati, J., Jaton-Ogay, K., Ave, P. \& Latgé, J. P. (1993a). Virulence of alkaline protease-deficient mutants of Aspergillus fumigatus. FEMS Microbiol Lett 106, 39-46.

Monod, M., Paris, S., Sanglard, D., Jaton-Ogay, K., Bille, J. \& Latgé, J. P. (1993b). Isolation and characterization of a secreted metalloprotease of Aspergillus fumigatus. Infect Immun 61, 40994104.

Monod, M., Léchenne, B., Jousson, O., Grand, D., Zaugg, C., Stöcklin, R. \& Grouzmann, E. (2005). Aminopeptidases and dipeptidyl-peptidases secreted by the dermatophyte Trichophyton rubrum. Microbiology 151, 145-155.

Monod, M., Jousson, O. \& Reichard, U. (2009). Aspergillus fumigatus secreted proteases. In Aspergillus fumigatus and Aspergillosis, pp. 87106. Edited by J.-P. Latgé \& W. J. Steinbach. Washington, DC: American Society for Microbiology.

O'Donoghue, A. J., Mahon, C. S., Goetz, D. H., O'Malley, J. M., Gallagher, D. M., Zhou, M., Murray, P. G., Craik, C. S. \& Tuohy, M. G. (2008). Inhibition of a secreted glutamic peptidase prevents growth of the fungus Talaromyces emersonii. J Biol Chem 283, 29186-29195.

Oda, K. (2004). Scytalidopepsin B. In Handbook of Proteolytic Enzymes, 2nd edn, pp. 219-221. Edited by A. J. Barrett, N. D. Rawlings \& J. F. Woessner. London: Elsevier.

Punt, P. J., Schuren, F. H., Lehmbeck, J., Christensen, T., Hjort, C. \& van den Hondel, C. A. (2008). Characterization of the Aspergillus niger $\operatorname{prt} T$, a unique regulator of extracellular protease encoding genes. Fungal Genet Biol 45, 1591-1599.

Reichard, U., Büttner, S., Eiffert, H., Staib, F. \& Rüchel, R. (1990). Purification and characterisation of an extracellular serine proteinase from Aspergillus fumigatus and its detection in tissue. J Med Microbiol 33, 243-251.

Reichard, U., Monod, M. \& Rüchel, R. (1995). Molecular cloning and sequencing of the gene encoding an extracellular aspartic proteinase from Aspergillus fumigatus. FEMS Microbiol Lett 130, 69-74.

Reichard, U., Monod, M., Odds, F. \& Rüchel, R. (1997). Virulence of an aspergillopepsin-deficient mutant of Aspergillus fumigatus and evidence for another aspartic proteinase linked to the fungal cell wall. J Med Vet Mycol 35, 189-196.

Reichard, U., Léchenne, B., Asif, A. R., Streit, F., Grouzmann, E., Jousson, O. \& Monod, M. (2006). Sedolisins, a new class of secreted proteases from Aspergillus fumigatus with endoprotease or tripeptidylpeptidase activity at acidic pHs. Appl Environ Microbiol 72, 17391748.

Rolland, S., Bruel, C., Rascle, C., Girard, V., Billon-Grand, G. \& Poussereau, N. (2009). $\mathrm{pH}$ controls both transcription and posttranslational processing of the protease BcACP1 in the phytopathogenic fungus Botrytis cinerea. Microbiology 155, 2097-2105.

Ronquist, F. \& Huelsenbeck, J. P. (2003). MrBayes 3: Bayesian phylogenetic inference under mixed models. Bioinformatics 19, 15721574.

Sasaki, H., Tanokura, M., Muramatsu, T., Nakagawa, A., Iwata, S., Hamaya, T., Takizawa, T., Kono, T. \& Takahashi, K. (1995). X-ray crystallographic study of a non-pepsin-type acid proteinase, Aspergillus niger proteinase A. Adv Exp Med Biol 362, 605-609.

Sharon, H., Hagag, S. \& Osherov, N. (2009). Transcription factor PrtT controls expression of multiple secreted proteases in the human pathogenic mold Aspergillus fumigatus. Infect Immun 77, 40514060.

Shevchenko, A., Wilm, M., Vorm, O. \& Mann, M. (1996). Mass spectrometric sequencing of proteins silver-stained polyacrylamide gels. Anal Chem 68, 850-858.

Sriranganadane, D., Waridel, P., Salamin, K., Reichard, U., Grouzmann, E., Neuhaus, J. M., Quadroni, M. \& Monod, M. (2010). 
Aspergillus protein degradation pathways with different secreted protease sets at neutral and acidic pH. J Proteome Res 9, 3511-3519.

Takahashi, K. (2004). Apergillopepsin II. In Handbook of Proteolytic Enzymes, 2nd edn, pp. 221-224. Edited by A. J. Barrett, N. D. Rawlings \& J. F. Woessner. London: Elsevier.

Tilburn, J., Scazzocchio, C., Taylor, G. G., Zabicky-Zissman, J. H., Lockington, R. A. \& Davies, R. W. (1983). Transformation by integration in Aspergillus nidulans. Gene 26, 205-221.

von Heijne, G. (1986). A new method for predicting signal sequence cleavage sites. Nucleic Acids Res 14, 4683-4690.
Wilm, M., Shevchenko, A., Houthaeve, T., Breit, S., Schweigerer, L., Fotsis, T. \& Mann, M. (1996). Femtomole sequencing of proteins from polyacrylamide gels by nano-electrospray mass spectrometry. Nature 379, 466-469.

Yabuki, Y., Kubota, K., Kojima, M., Inoue, H. \& Takahashi, K. (2004). Identification of a glutamine residue essential for catalytic activity of aspergilloglutamic peptidase by site-directed mutagenesis. FEBS Lett 569, 161-164.

Edited by: J. F. Ernst 